\title{
Outcomes of Ureteral Stent Placement for Hydronephrosis in Patients with Gynecological Malignancies
}

\author{
Saya Kurata Shohei Tobu Kazuma Udo Mitsuru Noguchi \\ Department of Urology, Faculty of Medicine, Saga University, Saga, Japan
}

\section{Key Words}

Gynecological malignancy $\cdot$ Hydronephrosis $•$ Ureteral stent

\begin{abstract}
Objective: We examined the outcomes of patients undergoing ureteral stent placement for hydronephrosis that occurred during treatment for gynecological malignancies. Materials and Methods: From January 2004 to December 2009, we enrolled 33 patients with 45 ureters undergoing ureteral stent placement for hydronephrosis which occurred during treatment for gynecological malignancies. We examined the outcomes of the patients after stent placement. Results: The causes of hydronephrosis were obstruction of the urinary tract by a tumor $(n=22)$, obstruction due to lymph node swelling $(n=6)$, ureteral stenosis after radiation therapy $(n=4)$, and others $(n=1)$. The ureteral stent was inserted into both ureters in 12 cases, and into one ureter in 21 cases. Ureteral stents were replaced 1-26 times during the observation period (median 3 times). Eighteen (40\%) ureteral stents were removed. The reasons for ureteral stent removal were hydronephrosis improvement (11 ureters, 24.4\%), a change to nephrostomy (cystectomy: 1 ureter, progression of ureteral stenosis: 2 ureters), renal atrophy (3 ureters), and ureteral dilatation (1 ureter). All of the cases in which ureteral stent withdrawal due to hydronephrosis improvement were cases in which the ureter was compressed by a tumor and were lower ureteral obstructions. Twenty-one patients (64\%) died due to cancer after stent placement. The periods from the first stent placement to death ranged from 1 to
\end{abstract}

\section{KARGER}

(C) 2016 S. Karger AG, Basel

Fax +4161306 1234

E-Mail karger@karger.com

www.karger.com
Accessible online at: www.karger.com/cur
58 months (median 18 months). Conclusion: Ureteral stent placement was associated with a poor prognosis in patients with gynecological malignancies. There were a few cases in which stent withdrawal became possible due to the improvement of hydronephrosis. In such cases, the withdrawal rate varied according to the cause and obstructive level.

Copyright $\odot 2016$ S. Karger AG, Basel

\section{Introduction}

Experience with ureteral stents was first reported in 1978 and stents were subsequently used for patients with malignancies that were causing external compression of the ureter [1]. Urologists perform ureteral stent placement to improve hydronephrosis. The actual incidence of malignant ureteral obstruction is unknown [2]. Malignant ureteral obstruction can arise from intrinsic urological malignancy, most commonly urothelial carcinoma, or extrinsically from other primary sources, most commonly gynecological or colorectal. For non-urological primary malignancies the obstruction is due to direct invasion, nodal disease, or involvement in an inflammatory process. Thus, many urologists are likely to perform ureteral stent placement to improve hydronephrosis in patients who were initially treated in other clinical departments. However, stent insertion may reduce quality of life (QOL) due to sharp pain, bladder stimulation, or 
hematuria and urologists should take care in considering stent placement. The authors of the present study took particular care in considering the indications for stent placement in cases caused by malignant tumors, with special consideration given to such aspects as the convalescence of the patient.

In our hospital, most often a ureteral stent is placed because of gynecological malignant tumors causing hydronephrosis. We think that aggressive medical treatment is often provided for progressive cancers with hydronephrosis because the age group of patients and radiotherapy are often provided for uterus preservation for early cancers. The cases in which ureteral stricture does not improve even if gynecological malignant tumors are cured should continue only after urological consultation for periodical ureteral stent exchange. There are patients that frequently need treatment for pain and acute pyelonephritis due to ureteral stent placement. Ureteral stent placement may affect everyday life in the long term. It is necessary for us to know the prognosis of patients after ureteral stent placement, and the future of the stent.

We therefore examined the clinical course of patients with gynecological malignant tumors in whom hydronephrosis occurred during medical treatment, and in which ureteral stent placement was performed.

\section{Materials and Methods}

We retrospectively examined the cases of 33 ureteral stent patients (45 ureters) in whom hydronephrosis occurred. All of the patients had undergone treatment for gynecological malignancies from January 2004 to December 2009. The reason why we used this period is that we wanted to observe them for more than 5 years. In our hospital, stenting is performed according to the following: 1) patients with symptomatic hydronephrosis, 2) patients with impaired renal function, and 3) patients with asymptomatic hydronephrosis in whom prognostic improvement can be expected with the cure of the underlying disease. We used the polyolefin type of ureteral stent for all patients. Ureteral stents were changed every 3-6 months. The degree of hydronephrosis was evaluated by renal ultrasonography or computed tomography. In these cases, we examined ureteral stent withdrawal, patient outcomes, and the harmful phenomena associated with stent placement.

\section{Results}

Patients of the present study were of 30-80 years of age (average 57 years). The observation period after stenting ranged from 1 to 122 months (median 28 months). We observed the survivors for more than 5 years. The observation period of less than 5 years was for patients who
Table 1. Characteristics of patients

\begin{tabular}{lc}
\hline Variable & Value \\
\hline Age (year), mean (range) & $57(30-80)$ \\
Follow up (month), mean (range) & $46(1-122)$ \\
Primary disease, n (\%) & \\
$\quad$ Uterine (cervical) cancer & $20(61)$ \\
$\quad$ Ovarian cancer & $9(27)$ \\
$\quad$ Uterine (corpus) cancer & $2(6)$ \\
$\quad$ Other & $2(6)$ \\
Cause of hydronephrosis, n (\%) & \\
$\quad$ Tumor & $22(67)$ \\
$\quad$ Lymph node & $6(18)$ \\
$\quad$ Radiation & $4(12)$ \\
Other & $1(3)$ \\
Inserted side, n $(\%)$ & $12(36)$ \\
$\quad$ Bilateral & $21(64)$ \\
$\quad$ Unilateral &
\end{tabular}

Table 2. Level of ureteral obstruction

\begin{tabular}{lllc}
\hline Variable & Upper & Middle & Lower \\
\hline Tumor $(\mathrm{n}=32)$ & 0 & 5 & 27 \\
Lymph node $(\mathrm{n}=7)$ & 4 & 3 & 0 \\
Radiation $(\mathrm{n}=5)$ & 1 & 1 & 3 \\
Other $(\mathrm{n}=1)$ & 0 & 0 & 1 \\
Total & $5(11 \%)$ & $9(20 \%)$ & $31(69 \%)$ \\
\hline
\end{tabular}

Table 3. Cases of stent withdrawal $(n=45)$

\begin{tabular}{ll}
\hline Variable & Ureter, $\mathrm{n}(\%)$ \\
\hline $\begin{array}{l}\text { Hydronephrosis improvement } \\
\text { Change to nephrostomy }\end{array}$ & $11(24.4)$ \\
$\quad$ Cystectomy & $1(2.2)$ \\
$\quad$ Progression of ureteral stenosis & $2(4.4)$ \\
Kidney atrophy & $3(6.7)$ \\
Ureteral dilatation & $1(2.2)$ \\
Total & $18(40)$ \\
\hline
\end{tabular}

died before 5 years. The causative diseases were cervical cancer $(n=20)$, ovarian cancer $(n=9)$, endometrial cancer $(n=2)$, other gynecological malignancies $(n=$ 2). Hydronephrosis occurred as a result of confinement of the ureter due to a tumor ( $n=22,32$ ureters), due to 


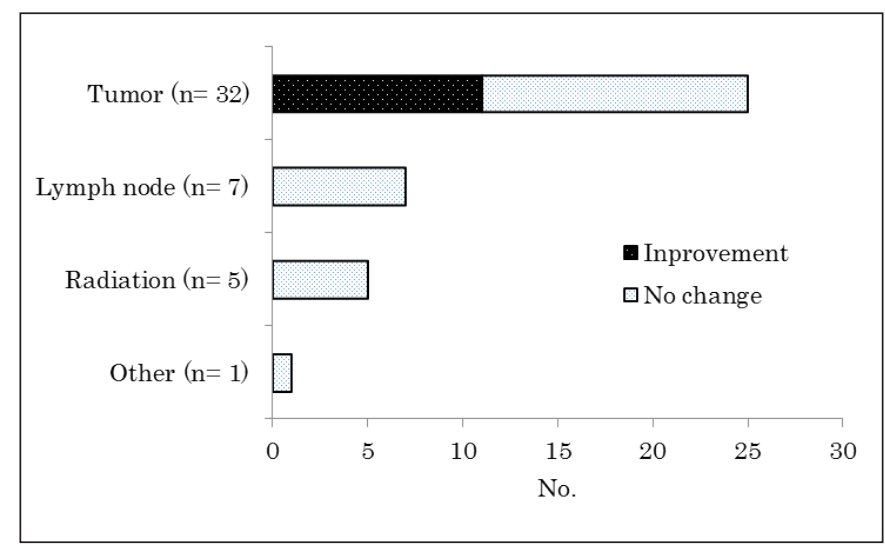

Fig. 1. All of the cases in which ureteral stent withdrawal was possible due to hydronephrosis improvement were cases in which the ureter was compressed by a tumor.

confinement ( $\mathrm{n}=6,7$ ureters) to lymphadenopathy, or ureteral stenosis after radiation therapy ( $\mathrm{n}=4,5$ ureters), and due to other causes ( $\mathrm{n}=1,1$ ureter). The insertion was on both sides in 12 cases and one side in 21 cases (table 1). Five ureters (11\%) had an upper level ureteral obstruction, 9 (20\%) had a mid ureteral obstruction, and $31(69 \%)$ had a lower ureteral obstruction (table 2).

The patients underwent stent placement 1-26 times (median 3 times) during the observation period. The complications that occurred during hospitalization that required invasive treatment were as follows: acute pyelonephritis $(n=4)$, stent confinement $(n=2)$, lithiasis adhesion $(\mathrm{n}=1)$, and stent migration $(\mathrm{n}=1)$.

Stent withdrawal was possible in 18 of 45 ureters (40\%) (table 3). Ureteral stent withdrawal was performed due to the improvement of hydronephrosis in 11 ureters (24.4\%), a change to nephrostomy in 3 ureters (6.7\%) (total cystectomy, 1 ureter, progression of ureteral stenosis, 2 ureters), kidney atrophy in 3 ureters (6.7\%), and after ureter dilation in 1 ureter $(2.2 \%)$. All of the cases in which ureteral stent withdrawal was possible due to hydronephrosis improvement were cases in which the ureter was compressed by a tumor (fig.1) and was a lower ureteral obstruction (table 4). In many cases the first stent was removed within approximately 1 year (range 28-1,589 days, average 393 days) (table 4). Only one of the patients who underwent stent withdrawal died. We changed from ureteral stent placement to nephrostomy to perform a total hysterectomy and total cystectomy for local recurrence after radiation therapy for cervical cancer in 1 ureter, and in 2 ureters, respectively, in which it was

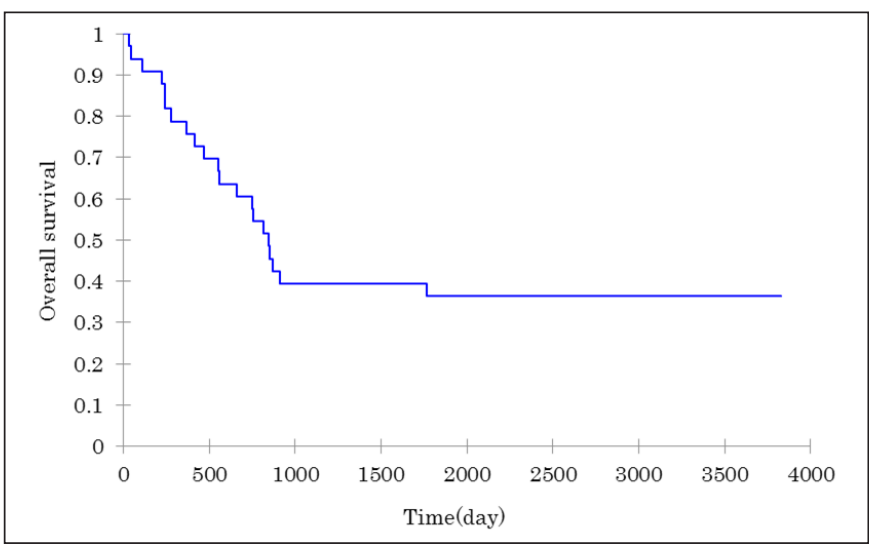

Fig. 2.Survival curves showing the time to death from the initial insertion.

necessitated by the progression of ureteral stenosis. In 3 ureters, kidney atrophy occurred after stenting and the stents were removed because stenting was not necessary to protect renal function. In these cases the stent was removed at 3, 81, and 69 months after stenting (table 5). In 1 case, ureteral stenosis caused by radiation treatment required ureteral dilation due to severe bladder stimulation symptoms after stent placement. In that case the patient underwent bilateral stent placement, and bilateral ureteral dilatation was performed. However, stent removal was only possible on one side.

Twenty-one patients (64\%) died after stent placement and $12(36 \%)$ survived. Death occurred within 1 year after stent placement in 7 cases $(21 \%)$. The outcomes according to the causes of hydronephrosis were as follows: tumor (death in 13 and survival in 9), lymph node swelling (death in 6 and survival in 0), radiation therapy (death in 1 and survival in 3), others (death in 1 and survival in 0). Ureteral stent placement was continued in 3 of the 12 survivors. In all 3 cases, this was due to ureteral stricture that occurred with radiotherapy (table 6). The time to death from the initial insertion ranged from 34 to 1,765 days (average 575 days) (fig. 2).

\section{Discussion}

Ureteral stent placement is frequently conducted to treat various pathological conditions that cause upper urinary tract obstruction. Stent placement is commonly used to treat hydronephrosis, which occurs due to conditions 
Table 4. Characteristics of ureteral stent withdrawal due to hydronephrosis improvement

\begin{tabular}{llllllr}
\hline Primary disease & Age (years) & Level & Treatment & Cause & Outcome & Placement period (day) \\
\hline Ovarian cancer & 73 & lower & surgery, chemo & tumor & no recurrence & 28 \\
Cervical cancer & 68 & lower & CCRT & tumor & no recurrence & 50 \\
Cervical cancer & 31 & lower & CCRT & tumor & death & 117 \\
Ovarian cancer & 43 & lower & surgery, chemo & tumor & no recurrence & 180 \\
Cervical cancer & 64 & lower & CCRT & tumor & no recurrence & 255 \\
Cervical cancer & 44 & lower & CCRT & tumor & no recurrence & 353 \\
Cervical cancer & 44 & lower & CCRT & tumor & no recurrence & 353 \\
Corpus cancer & 68 & lower & surgery, chemo & tumor & no recurrence & 378 \\
Corpus cancer & 68 & lower & surgery, chemo & tumor & no recurrence & 385 \\
Cervical cancer & 58 & lower & CCRT & tumor & no recurrence & 637 \\
Cervical cancer & 51 & lower & CCRT & tumor & no recurrence & 1589 \\
\hline
\end{tabular}

CCRT $=$ Concurrent chemoradiotherapy

Table 5. In 3 ureters, kidney atrophy occurred after stenting and the stents were removed because stenting was not necessary to protect renal function

\begin{tabular}{rlllllr}
\hline Exchange times & Age (years) & Level & Primary disease & Cause & Outcome & Placement (month) \\
\hline 1 & 33 & Lower & cervical cancer & tumor & death & 3 \\
22 & 64 & Lower & cervical cancer & tumor & no recurrence & 69 \\
18 & 32 & Lower & cervical cancer & tumor & no recurrence & 81 \\
\hline
\end{tabular}

Table 6. Outcomes according to the causes of hydronephrosis

\begin{tabular}{lrl}
\hline Variable & Death & Alive \\
\hline Tumor $(\mathrm{n}=22)$ & 13 & 9 (placement 0) \\
Lymph node $(\mathrm{n}=6)$ & 6 & 0 \\
Radiation $(\mathrm{n}=4)$ & 1 & 3 (placement 3) \\
Other $(\mathrm{n}=1)$ & 1 & 0 \\
Total & 21 & 12 \\
\hline
\end{tabular}

other than urological diseases. Our hospital has decided on the indications for ureteral stent placement to treat upper urinary tract obstruction due to cancer progression. Stent placement is indicated in patients with symptomatic hydronephrosis, patients with renal dysfunction, or in patients with asymptomatic hydronephrosis who have an Eastern Cooperative Oncology Group performance status of $0-1$ and in whom stenting can be expected to improve the prognosis of the primary disease.

Ureteral Stent Placement for Hydronephrosis in Patients with

Gynecological Malignancies
However, there was a report indicating that $85.2 \%$ of patients with symptomatic hydronephrosis showed symptom improvement after stent placement [3]. The failure rate of ureteral stent placement was reported to be $16-58 \%$ [4]. Nephrostomy may be required to remove the urinary obstruction. However, due to fears of reducing the patient's QOL, ureteral stent placement is often attempted in place of nephrostomy. However, it should be considered that ureteral stent placement can result in a reduced QOL with regard to urinary frequency, the feeling of residual urine, the feeling of urinary urgency, and a feeling of incongruity in the abdominal region. Joshi et al. [3] conducted an investigation on the prevalence of stent-related conditions that affected the QOL of patients who underwent ureteral stent placement. They reported that urination symptoms occurred in $76 \%$ of their study population, while pain that required the use of analgesics occurred in $70 \%$, and was due to decreased activity in $42 \%$. We therefore carefully considered the indications for stent placement from the point of view of QOL. The

Curr Urol 2016;10:126-131 
present study only included patients with relatively severe complications that required hospitalization and invasive treatment. The frequency of complications such as a sense of incongruity or the inflammation of the bladder would be much higher if we included the cases of patients who were being treated at outpatient departments. In 2001, the Ureteric Stent Symptoms Questionnaire was proposed to evaluate the QOL and symptoms of ureteral stent recipients [5, 6]. Acute pyelonephritis and calculi formation that are present in most of the cases with complications may easily occur when stents are placed for a long period of time [7]. For this reason, an exchange period of 2-4 months is favored [8]. We aim to change stents every 3 months in our hospital. However, cases in which repeat obstruction occurs may require a more rapid exchange. One more unfortunate late complication of stenting is a "lost" or "forgotten" stent [9]. It is due to human factors and, thus is entirely preventable. As discussed, increasing dwell times lead to increased risk of encrustation, often making removal more difficult and dangerous. Urologists should have an active role in preventing this complication.

The success rate of initial retrograde ureteral stent placement in cases of malignant ureteral obstruction was reported as 72.5 to $79 \%[10,11]$. Factors reported as predictors of stent failure were baseline serum creatinine, no treatment after retrograde ureteral stent placement, gross tumor invasion noted at cystoscopy, the degree of hydronephrosis, and male gender [12-15].

The prognosis of patients with cancer progression that results in upper urinary tract obstruction is poor. One report indicated that the survival period after the occurrence of obstruction occurrence is 3-7 months [16]. Zadra et al. [17] showed the worst outcomes in patients with malignant ureteral obstruction secondary to metastatic breast cancer (3.74 months, range $0-11$ months) compared to malignant ureteral obstruction secondary to other malignancies such as cervical cancer (11.29 months, range 0-60 months). Disease stage or grade (other than metastases from breast cancer), age, and degree of renal impairment have no effect on prognosis. Izumi et al. [10] considered a series of patients with gynecological and colorectal cancer with an overall median survival of 228 days. Unfavorable prognostic factors of overall survival were no treatment after indwelling retrograde ureteral stent placement $(\mathrm{p}=0.023)$ and a serum creatinine before indwelling retrograde ureteral stent placement of 1.2 $\mathrm{mg} / \mathrm{dl}$ or greater $(\mathrm{p}=0.016)$. Our study showed death occurred at 1-58 months (average 19 months, median 18 months) after ureteral stent replacement and $21 \%$ of the patients in the present study died within 1 year of stent placement. The survival curve indicated that most deaths occurred within 3 years (fig. 2). Although ureteral stent replacement is performed to improve upper urinary tract obstruction, when 5, 6, and 7Fr urinary stents are placed in the normal urinary tract, the urinary flow is reported to decrease to 83,61 and $58 \%$, respectively [18]. In other words, ureteral stent replacement is not a permanent solution to renal dysfunction. In the present study 3 cases required ureteral stent removal due to renal atrophy. In 1 case with renal atrophy after the initial placement, removal was performed 3 months later, and in the other two, stent withdrawal was done after 69 and 81 months (during which time stents were exchanged) (table 5).

When we perform ureteral stent placement, the patient may ask whether or not stent withdrawal will be possible in the future. There are cases in which stent withdrawal is not possible, even if the primary disease heals. In the present study, this withdrawal was possible in 11 out of 32 ureters due to the improvement of hydronephrosis. In all these cases hydronephrosis was caused by a tumor (table 3). In most of the cases where withdrawal was possible, we were able to remove the stent within approximately one year (table 4). None of the cases of radiation-related hydronephrosis showed improvement, and in all of the surviving patients who had undergone radiotherapy, stent placement was continued (table 6). Thus, the possibility of ureteral stent withdrawal depends on the cause. In approximately one-third of cases in which the cause is a tumor, stent withdrawal becomes possible after the medical treatment of the primary disease. Stents are often removed within 1 year, and the withdrawal rate decreases thereafter. In patients with stenosis associated with radiotherapy, stent withdrawal after the improvement of hydronephrosis is difficult. In many cases with lymphadenopathy, we cannot expect stent withdrawal to be possible due to the poor prognosis of the primary disease. Ureteral stricture may progress with the condition. In 2 urinary tracts $(4.4 \%)$ ureteral stent insertion was difficult, and we performed nephrostomy. Chung et al. [19] reported that when they managed ureteral stricture due to malignant tumors with a ureteral stent, management of the stenting became impossible, and $56 \%$ of patients underwent percutaneous nephrostomy within 11 months.

There is no consistent opinion about stent placement, and the adaptation is left to the judgment of the doctor. It is necessary for us to provide a sufficient explanation that stent placement is associated with a decreased QOL, the likelihood of nephrostomy, and the likelihood of stent withdrawal if stent placement is requested rather than to 
simply perform stent placement because it is requested. It is necessary to take responsibility for the insertion of the stent until the end of treatment. The long-term nature of the treatment means that the indications should be considered.

\section{References}

1 Finney RP: Experience with new double J ureteral catheter stent. J Urol 1978;120:678681.

2 Kouba E, Wallen EM, Pruthi RS: Management of ureteral obstruction due to advanced malignancy: optimizing therapeutic and palliative outcomes. J Urol 2008;180:444-450.

3 Joshi HB, Stainthorpe A, MacDonagh RP, Keeley FX Jr, Timoney AG, Barry MJ: Indwelling ureteral stents: evaluation of symptoms, quality of life and utility. J Urol 2003; 169:1065-1069.

4 Wong LM, Cleeve LK, Milner AD, Pitman AG: Malignant ureteral obstruction: outcome after intervention. Have things changed? J Urol 2007; 178:178-183.

5 Joshi HB, Newns N, Stainthorpe A, MacDonagh RP, Keeley FX Jr, Timoney AG: Ureteral stent symptom questionnaire: development and validation of a multidimensional quality of life measure. J Urol 2003;169: 1060-1064.

6 Joshi HB, Stainthorpe A, Keeley FX Jr, MacDonagh R, Timoney AG: Indwelling ureteral stents: evaluation of quality of life to aid outcome analysis. J Endourol 2001;15:151-154.

\section{Conclusion}

In patients with gynecological malignancies, ureteral stent placement has a poor prognosis. Stent withdrawal with hydronephrosis improvement was only possible in a relatively small number of patients and the withdrawal rate varied with the cause.
7 Duvdevani M, Chew BH, Denstedt JD: Minimizing symptoms in patients with ureteric stents. Curr Opin Urol 2006;16:77-82.

8 Singh I, Gupta NP, Hemal AK, Aron M, Seth A, Dogra PN: Severely encrusted polyurethane ureteral stents: management and analysis of potential risk factors. Urology 2001;58: 526-531.

9 Fiuk J, Bao Y, Calleary JG, Schwartz BF, Denstedt JD: The use of internal stents in chronic ureteral obstruction. J Urol 2015;193: 1092-1100.

10 Izumi K, Mizokami A, Maeda Y, Koh E, Namiki M: Current outcome of patients with ureteral stents for the management of malignant ureteral obstruction. J Urol 2011;185: 556-561.

11 Kanou T, Fujiyama C, Nishimura K, Tokuda Y, Uozumi J, Masaki Z: Management of extrinsic malignant ureteral obstruction with urinary diversion. Int J Urol 2007;14:689692.

12 Chung SY, Stein RJ, Landsittel D, Davies BJ, Cuellar DC, Hrebinko RL, Tarin T, Averch TD: 15-Year experience with the management of extrinsic ureteral obstruction with indwelling ureteral stents. J Urol 2004;172: 592-595.
13 Jeong IG, Han KS, Joung JY, Seo HK, Chung $\mathrm{J}$ : The outcome with ureteric stents for managing non-urological malignant ureteric obstruction. BJU Int 2007;100:1288-1291.

14 Ganatra AM, Loughlin KR: The management of malignant ureteral obstruction treated with ureteral stents. J Urol 2005;174:2125-2128.

15 Wenzler DL, Kim SP, Rosevear HM, Faerber GJ, Roberts WW, Wolf JS Jr: Success of ureteral stents for intrinsic ureteral obstruction. J Endourol 2008:22:295-299.

16 Ganatra AM, Loughlin KR: The management of malignant ureteral obstruction treated with ureteral stent. J Urol 2005;174:2125-2128.

17 Zadra JA, Jewett MA, Keresteci AG, Rankin JT, St Louis E, Grey RR, Pereira JJ: Nonoperative urinary diversion for malignant ureteral obstruction. Cancer 1987;60:1353-1357.

18 Burgos Revilla FJ, Sáez Garrido JC, Vallejo Herrador J, Lovaco Castellano F, Del Hoyo Campos J: Hydrodynamic behavior of endouroligic catheterts. Arch Esp Urol 1995;48: 627-636.

19 Feuer GA, Fruchter R, Seruri E, Maiman M, Remy JC, Boyce JG: Selection for percutaneous nephrostomy in gynecologic cancer patients. Gynecol Oncol 1991;42:60-63. 